Revue musicale OICRM

\title{
Compte rendu du douzième Forum international des jeunes compositeurs
}

\section{Philippe Béland}

Volume 2, numéro 2, 2015

Une relève

URI : https://id.erudit.org/iderudit/1060138ar

DOI : https://doi.org/10.7202/1060138ar

Aller au sommaire du numéro

Éditeur(s)

OICRM

ISSN

2368-7061 (numérique)

Découvrir la revue

Citer cette note

Béland, P. (2015). Compte rendu du douzième Forum international des jeunes compositeurs. Revue musicale OICRM, 2(2), 197-203.

https://doi.org/10.7202/1060138ar
Résumé de l'article

Le douzième Forum international des jeunes compositeurs du Nouvel Ensemble Moderne s'est déroulé à la Faculté de musique de l'Université de Montréal du 1er au 21 novembre 2014. Pour cette nouvelle édition, huit jeunes compositeurs provenant des quatre coins de la planète ont composé une œuvre d'une dizaine de minutes pour cet ensemble, qu'ils ont travaillé en présence des musiciens et de sa chef, Lorraine Vaillancourt. Ce texte présente également une courte analyse de la pièce gagnante, Utrecht, du compositeur mexicain Francisco Castillo Trigueros, présentant la forme de l'œuvre, sa structure ainsi que les différents matériaux musicaux la composant. 


\title{
Compte rendu du douzième Forum international des jeunes compositeurs

\author{
Philippe Béland
}

\begin{abstract}
Résumé
Le douzième Forum international des jeunes compositeurs du Nouvel Ensemble Moderne s'est déroulé à la Faculté de musique de l'Université de Montréal du $1^{\mathrm{er}}$ au 21 novembre 2014. Pour cette nouvelle édition, huit jeunes compositeurs provenant des quatre coins de la planète ont composé une œuvre d'une dizaine de minutes pour cet ensemble, qu'ils ont travaillé en présence des musiciens et de sa chef, Lorraine Vaillancourt. Ce texte présente également une courte analyse de la pièce gagnante, Utrecht, du compositeur mexicain Francisco Castillo Trigueros, présentant la forme de l'oeuvre, sa structure ainsi que les différents matériaux musicaux la composant.
\end{abstract}

Mots clés: analyse musicale ; Forum 2014; Nouvel Ensemble Moderne; Francisco Castillo Trigueros; Utrecht.

\begin{abstract}
The $12^{\text {th }}$ edition of the Forum international des jeunes compositeurs, by the Nouvel Ensemble Moderne (NEM), took place at the Music Faculty of the University of Montreal from november $1^{\text {st }}$ to the $21^{\text {st }}, 2014$. For this new edition, eight young composers from all around the world composed a piece of 10-15 minutes in duration for the NEM, and worked with the musicians and their conductor, Lorraine Vaillancourt. This paper also presents a short analysis of the winner piece, Utrecht, by the mexican composer Francisco Castillo Trigueros, focusing on the form, structure and musical elements.
\end{abstract}

Keywords: Forum 2014; Music Analysis; Nouvel Ensemble Moderne; Francisco Castillo Trigueros; Utrecht. 
Le douzième Forum international des jeunes compositeurs s'est déroulé à la salle Claude-Champagne de la Faculté de musique de 1'Université de Montréal du $1^{\text {er }}$ au 21 novembre dernier. Depuis sa première édition, en 1991, ce Forum permet à de jeunes compositeurs, âgés de moins de 35 ans et provenant des quatre coins de la planète, de parfaire leur art en écrivant une pièce d'une dizaine de minutes pour le Nouvel Ensemble Moderne, dirigé depuis ses débuts par Lorraine Vaillancourt. Ces œuvres sont répétées pendant les trois semaines du Forum pour ensuite être interprétées devant public et soumises au même jury du Forum qui en a sélectionné les participants.

Les participants à cette douzième édition du Forum-nommément Artur Akshelyan (Arménie), Matei Gheorghiu (Roumanie), Dominique Lafortune (Canada), Theocharis Papatrechas (Grèce), Matthew Ricketts (Canada), Francisco Trigueros (Mexique), Nina C. Young (États-Unis) et Itzam Zapata (Mexique) - ont été choisis par un jury composé cette année des compositeurs John Rea (Canada), Allain Gaussin (France), Carlos Sanchez-Gutierrez (Mexique), du musicologue Frans Van Rossum (Pays-Bas) ainsi que de la directrice artistique Lorraine Vaillancourt (Canada).

Cette édition a permis à ces huit jeunes compositrices et compositeurs de partager avec le public montréalais leur vision de la musique non seulement à l'occasion des répétitions, des deux concerts, mais aussi au cours d'un lunch-causerie où chacun a pu présenter son parcours musical et personnel et répondre aux questions du public. L'expérience des membres du Forum s'est également inscrite dans le milieu académique de la Faculté de musique de 1'Université de Montréal par leur participation active aux cours d'Analyse et de Techniques modernes de composition dans le cadre desquels ils ont pu présenter une analyse de leur œuvre composée pour ce concours.

Le Forum offre un cadre précieux à des talents émergents pour bénéficier d'une collaboration unique et personnalisée tant avec des instrumentistes qu'avec la direction d'un ensemble autour d'une composition inédite. Chaque pièce est répétée pendant neuf heures, ce qui permet aux compositeurs de revenir dans le détail de leur travail et de le modifier au besoin en bénéficiant d'une écoute engagée et professionnelle. Le compositeur Francisco Castillo Trigueros témoigne ainsi de son expérience :

Travailler avec Lorraine et le NEM était incroyablement spécial. Il s'agit, et de loin, de l'ensemble qui a consacré le plus de temps et d'énergie à ma pièce. Ils ont pris le soin de travailler chaque détail, étaient flexibles aux changements que j'apportais aux répétitions et se consacraient entièrement à mon œuvre (ainsi qu'à celles des autres compositeurs). Lors des répétitions, j'ai senti que ma première esquisse comportait des faiblesses au niveau des proportions de la forme et j'y ai apporté des modifications. Heureusement, la structure du Forum permet ces ajustements. [...] Lorraine a été particulièrement utile. Elle a cru fermement à la pièce depuis le début, ce qui était très important pour moi. Elle était également disponible pour discuter des forces et faiblesses de ma musique et de ce qui pourrait être fait pour l'améliorer. [...] Je connaissais sa réputation de direction d'ensemble, auparavant, mais mes attentes ont été dépassées par son travail incroyable (Francisco Castillo Trigueros en réponse aux questions de l'auteur). 
À l'issue des deux concerts, le jury s'est réuni et au terme de ses délibérations a annoncé son palmarès attribuant le premier prix à Utrecht de Francisco Trigueros (Mexique) ainsi que le deuxième prix ex aequo aux pièces Tushpuea et Flatline des compositeurs Artur Akshelyan (Arménie) et Matthew Ricketts (Canada). Le coup de cœur du public a quant à lui été attribué, également ex aequo, à The Poetry of Negation et Sepium des compositeurs Itzam Zapata (Mexique) et Dominique Lafortune (Canada). Je me propose ici de présenter une courte analyse de la pièce qui a emporté le premier prix de ce douzième Forum.

\section{Francisco Castillo Trigueros}

Né en 1983, Francisco Castillo Trigueros est un compositeur et artiste sonore mexicain qui vit maintenant à Chicago. Il a étudié la théorie et la composition à la Rice University (Texas), au Conservatorium van Amsterdam et poursuit actuellement son doctorat à l'Université de Chicago. Trigueros a reçu de nombreuses distinctions, dont un BMI Student Composer Award, deux mentions honorables au Morton Gould Composer Awards ainsi que trois nominations pour le Gaudeamus Music Prize. Sa musique inclut souvent des dispositifs électroniques ainsi que des instrumentations et interprètes appartenant à d'autres cultures musicales.

Utrecht a été composée pour le Nouvel Ensemble Moderne (orchestre de chambre de quinze musiciens) et dure environ treize minutes. Le titre de cette œuvre est une dédicace à la ville des Pays-Bas où a séjourné Trigueros, en visite chez des amis, pour la composer. Il ne s'agit pas d'une musique à programme, la pièce consistant plutôt en une juxtaposition de divers matériaux musicaux contrastants.

Cette idée de juxtaposition fait suite à une pièce antérieure du compositeur, Resti, œuvre composée en collaboration avec le vidéaste Marco G. Ferrari. Il s'agit d'une création pour orchestre de chambre, vidéo et électronique. Dans cette dernière œuvre, Castillo explique qu'il a voulu créer une structure musicale extrêmement fragmentée, incorporant plusieurs matériaux diversifiés, afin de suivre la poésie du vidéo. Pour Utrecht, le compositeur a tenté de reproduire le même effet musical, mais cette fois-ci sans le visuel. Ces différents matériaux contrastés sont les suivants :

1. Courte séquence de sons de cloches (harmoniques de piano) qui subit des transformations et des distorsions. Agit à la fois comme un générateur de nouveaux matériaux ainsi que comme un pôle autour duquel les autres matériaux gravitent autour.

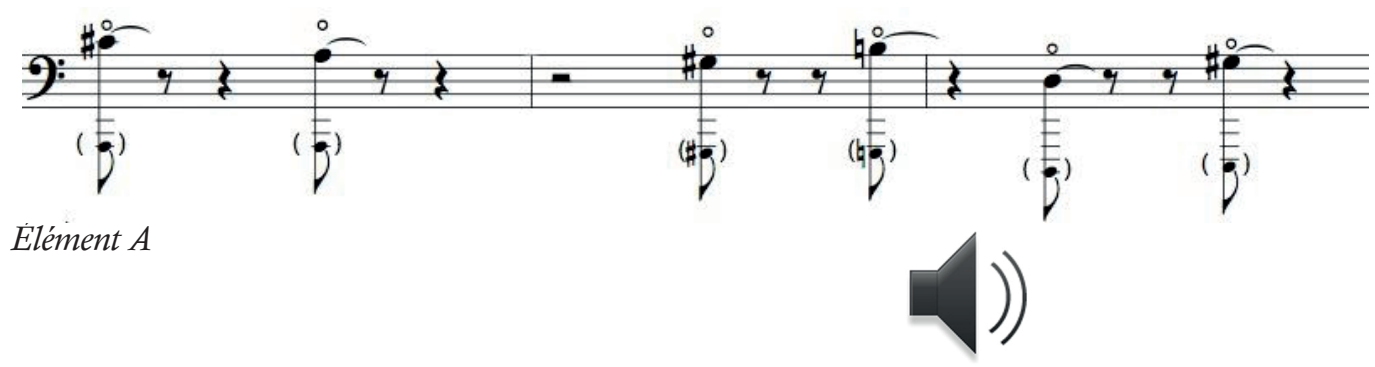


2. Progression harmonique de trois accords pianississimo aux cordes (avec microtonalité), basson et cuivres graves, ponctués par quatre coups rapides sur la grosse caisse (seul rôle de cet instrument dans la pièce).

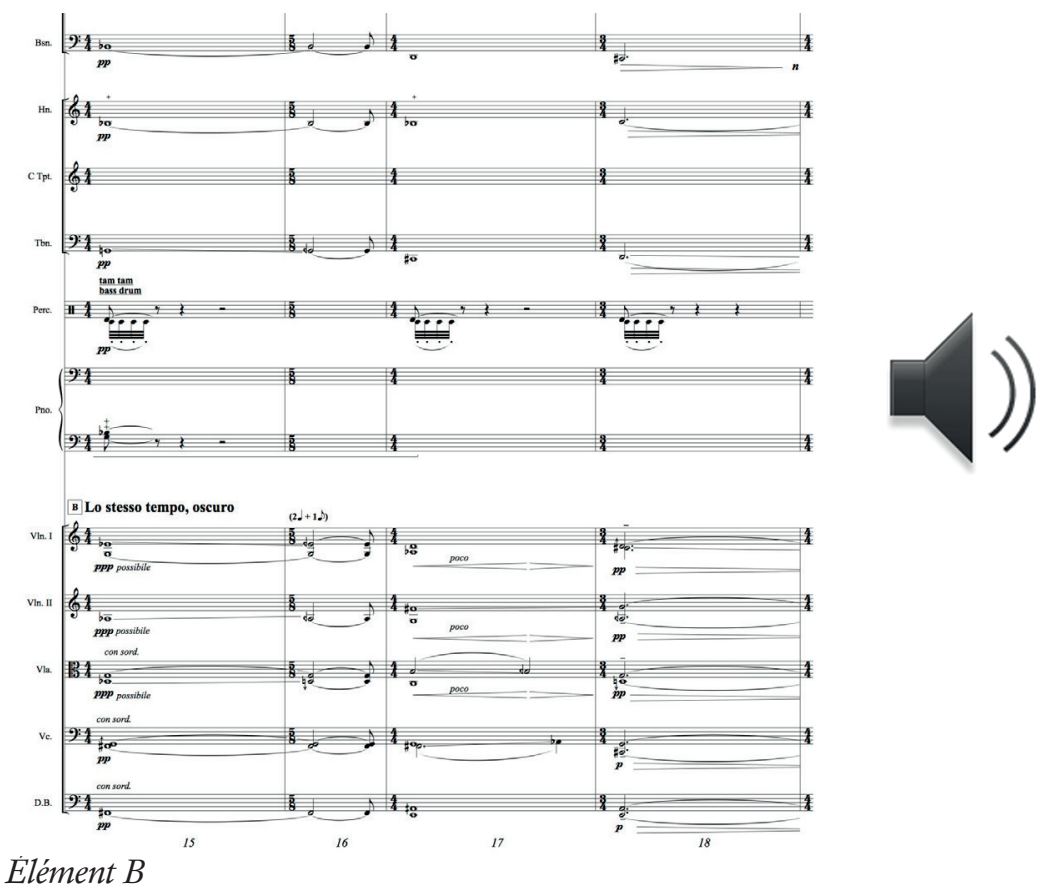

3. Gestes musicaux sur une seule note ou de bruit, suivant des courbes d'ondes, ressemblantes à des vagues.

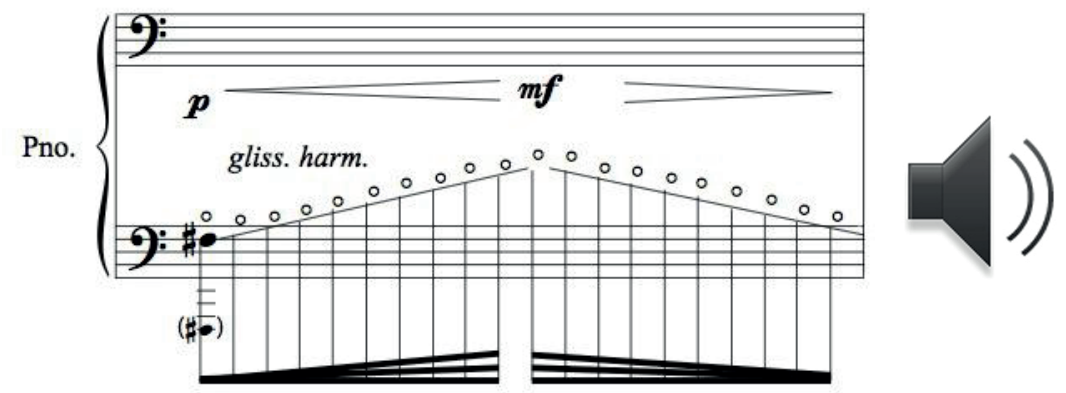

Élément $C 1$

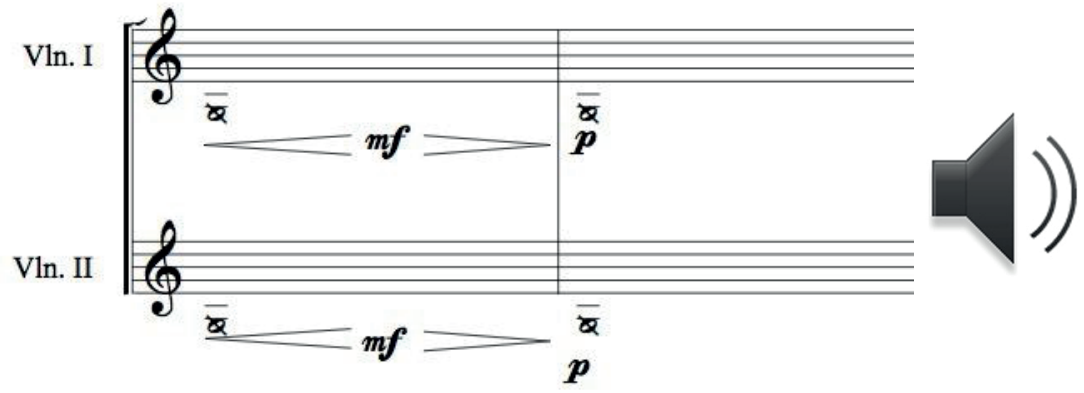

Élément $C 2$ 
4. Gestes violemment percussifs ascendants.

Vc.
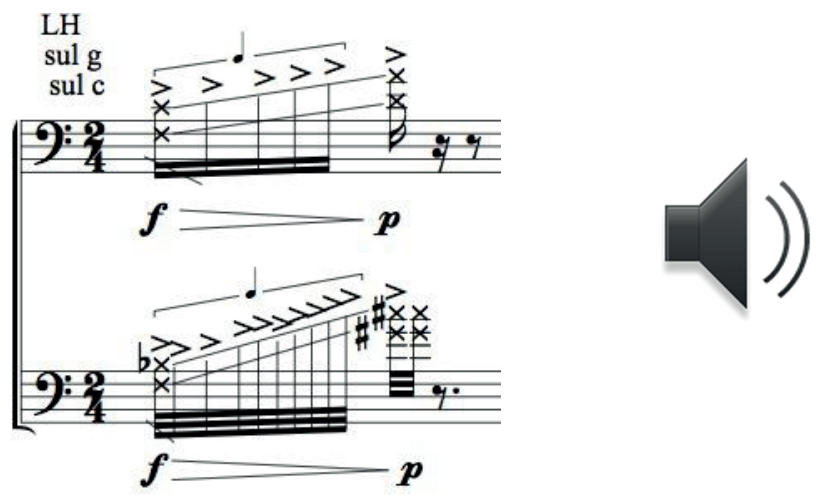

Élément $D$

Ces différents éléments sont présentés en alternance et les différentes juxtapositions permettent de varier la couleur individuelle de ces derniers. Ces idées sont contractées et étirées, selon les vagues d'énergies qui façonnent la musique. Bien qu'assez fragmentées (les idées sont très clairement délimitées), on retrouve six grandes sections qui regroupent ensemble ces différents éléments.

\begin{tabular}{|c|c|c|}
\hline Sections & Durée (min) & Éléments \\
\hline $\mathbf{1}$ & 4 & A - B \\
\hline $\mathbf{2}$ & 1 & C \\
\hline $\mathbf{3}$ & 1,75 & A - B - C \\
\hline $\mathbf{4}$ & 2 & D \\
\hline $\mathbf{5}$ & 2,5 & Mélange des différents éléments \\
\hline $\mathbf{6}$ & 2 & C \\
\hline
\end{tabular}

L'élément A ouvre la pièce, énoncé seulement par le piano. L'utilisation d'harmoniques au piano vient simuler des sons de cloches. Bien que de longueur différente à chaque itération, la cellule caractéristique de cet élément (trois premières notes descente d'une tierce majeure, suivie d'une seconde mineure) sera répétée plusieurs fois. Après deux itérations du piano, la séquence mélodique est reprise avec les cordes et bois en arrière-plan qui ajoutent une résonance et de l'ornementation. Ce fond agit comme une réverbération un peu distordue, qui se complexifie à chaque apparition. Cette résonance en arrière-plan se fige pour créer l'élément B (1'accord est cependant différent). La première section consiste en une alternance de longueur inexacte des éléments A et B. L'élément A évolue progressivement en se complexifiant alors que l'élément B est identique lors de sa reprise, excepté qu'il est bissé.

Les harmoniques de piano reviennent au début de la seconde section en introduisant l'élément $C$, qui consiste en de courtes vagues d'énergie. Dans ce cas-ci, plutôt que de 
s'en tenir à l'octave, le compositeur demande au pianiste un glissando d'harmonique sur une fondamentale. Nous retrouvons la couleur de cloches de l'élément A, mais avec un motif tout à fait différent. En réponse à ce geste, qui suit sensiblement une onde sinusoïdale, les cordes glissent également en harmonique pour faire ressortir différents partiels. Chaque instrument suit une courbe qui évolue à un rythme unique. L'élément $\mathrm{C}$ est celui qui subit le plus de variations tout au long de la pièce. En plus du geste ascendant du spectre harmonique mentionné ci-dessus, nous retrouvons également des courbes de volumes en soufflet chez les vents. Une technique couramment utilisée par les cordes dans cette œuvre consiste à jouer l'archet sur le pont de l'instrument, provoquant un son bruité sans hauteur perceptible. À deux reprises dans la pièce, on retrouve également ce geste en soufflet de bruit (voir exemple 2), qui répond au tam-tam. Ce geste se retrouve également à la fin de la pièce. Cependant, chaque instrument suit un rythme indépendant, créant un déséquilibre - une distorsion - de l'ensemble.

L'élément D est le plus contrastant et se trouve dans sa propre section de l'œuvre. Alors que la pièce est généralement assez douce et éthérée, l'élément $\mathrm{D}$ introduit un geste percussif rapide à tout l'ensemble. Chaque instrument utilise un mode de jeux permettant d'obtenir un son bruité, sans hauteur perceptible. Le changement de couleur, de rythme et de texture (un seul plan) contribue à ce contraste frappant. Cette section fait alterner deux variantes rythmiques et morphologiques de cette idée. L'utilisation de courbes bruitées est une évolution de l'élément C. Cependant, au lieu de vagues ascendantes et descendantes (sinusoïdale), les gestes suivent une seule direction (onde en dent de scie).

La cinquième section vient juxtaposer des versions courtes de chaque élément, avec l'élément $C$ en proéminence. Tout comme le parcours typique où l'on confronte différentes idées, l'on présente séparément chaque idée pour ensuite les faire confronter les unes contre les autres. Cette section vient jouer ce rôle. Cependant, plutôt que de chercher une grande confrontation, nous retrouvons plutôt une simple juxtaposition, comme peut en témoigner un tempo plus lent, des nuances plus douces et de plus grandes pauses entre les phrases.

L'élément A, tout comme les autres éléments, revient de façon modifiée. Nous ne retrouvons que deux ou trois notes de la séquence, généralement présentée conjointement par le trombone et le basson. Le piano ne joue presque plus, son rôle étant réservé principalement pour des éléments percussifs, à l'exception d'une reprise du glissando d'harmoniques qui revient à fin de l'œuvre.

De façon générale, il s'agit d'une pièce épurée où les courtes phrases sont clairement délimitées. Il est à noter que malgré la commande qui ne permettait pas au compositeur d'inclure un dispositif électronique ou bien d'instruments provenant d'autres cultures, on retrouve tout de même certaines sonorités inspirées par ceux-ci. Par exemple, il est demandé à la flûtiste tout au long de l'œuvre de jouer de la flûte basse, qui peut rappeler certaines flûtes telles que le shakuhachi. Le son des harmoniques du piano ainsi que le mode mélodique utilisé ont un côté orientalisant.

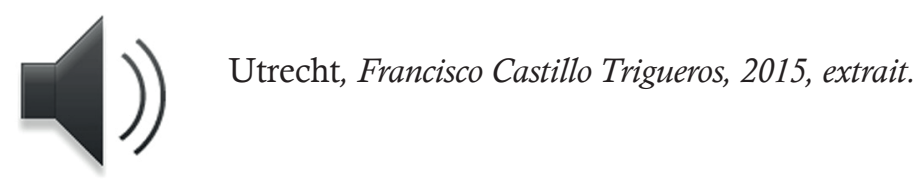


L'édition 2014 du Forum du Nouvel Ensemble Moderne a permis de mettre en lumière huit jeunes compositeurs de la relève. Plusieurs créateurs importants sont passés par cet événement incontournable, que l'on pense à Jimmie Leblanc, Brian Cherney, Javier Torres et plusieurs autres. L'expérience du Forum est très formatrice pour ces jeunes compositeurs et le sera de nouveau en 2016 pour la treizième édition du Forum international des jeunes compositeurs.

Pour en savoir plus : www.lenem.ca ; http://franciscocastillotrigueros.com/. 